\title{
Bending Motion in Split Flagella of Chlamydomonas
}

\author{
Shogo Nakamura and Ritsu Kamiya \\ Institute of Molecular Biology, Faculty of Science, Nagoya University, Nagoya \\ 464, Japan
}

ABSTRACT. Flagellar motility was studied in detergent-treated cells of Chlamydomonas with a dark-field microscope equipped with high-powered illumination. Flagella tended to split into two or three fibers in the presence of ATP and detergent. Some of split fibers showed oscillatory bending motion.

Movement of the $9+2$ system is based on active sliding between the outer doublet microtubules $(6,7,8)$, but the mechanism by which the sliding force is converted to an oscillatory bending motion is not clear. Thus we investigated to what extent the integrity of an axonemal structure is neccessary for the generation of bending force. We describe here some novel observations on detergent-extracted models of Chlamydomonas, and show that an incomplete portion of an axoneme originating from the splitting of a flagellum retains the ability to propagate bending waves.

A wild-type strain of Chlamydomonas reinhardtii C-239 (BIU-90, $\mathrm{mt}^{-}$) (a stock preserved in the Institute of Applied Microbiology, University of Tokyo) was grown by the method of Sager and Granick (5). Cell models were prepared by extraction with a non-ionic detergent, as described by Allen and Borisy (1). The extracting solution consisted of $10 \mathrm{mM}$ HEPES (N-2-hydroxyethyl-piperazine- $\mathrm{N}^{\prime}$-2-ethane sulfonic acid), $5 \mathrm{mM} \mathrm{MgSO}_{4}, 0.5 \mathrm{mM}$ mercaptoethanol, $0.5 \mathrm{mM}$ EDTA, $2 \%$ polyethylene glycol and $0.03 \%$ Nonidet $\mathrm{P} 40$. The $\mathrm{pH}$ value of this solution was adjusted to 8.1. We preferred observing axonemes at various stages of extraction, therefore reactivation of the extracted model was performed simultaneously with extraction, by adding $1 \mathrm{mM}$ ATP to the extracting solution. The behavior of axonemes during extraction and reactivation was monitored with a dark-field microscope equipped with highpowered illumination. Details of this optical system and of the techniques used have been described previously (3). With this system we could observe individual microtubules (4). The microscopic image was usually recorded by a TV-camera equipped with a built-in image-intensifier (CTC-9000, Ikegami Tsushinki Co., Tokyo) and by a video recorder, as this combination has been effective for recording the image of individual bacterial flagella (Hotani; unpublished results). Cinematography was sometimes used instead of the TV-method, although the microscopic image of the fine bundles of the microtubules was too dim to be recorded by this method.

When cells were transferred to an ATP-free extracting solution, all the flagella instantly stopped beating, and within a few minutes, some flagella became detached from the cell body. When $1 \mathrm{mM}$ ATP was present, many of the attached and detached flagella were beating even $30 \mathrm{~min}$ after the onset of extraction. The beating frequency had a maximum of about 4 beats/sec. During prolonged observation of the specimens in the presence of ATP and the detergent, for about $10 \mathrm{~min}$, some axonemes attached to the cell bodies became split in two or three pieces from the distal end. The frayed 
portion increased with time. These observations agreed with the results of previous authors $(1,9)$.

We found that many of these split axonemes still exhibit bending motion (Fig. 1). Figure 1 shows an axoneme which was split into two groups of microtubules of uneven width; the thicker one beating with a frequency of $1.3 \mathrm{beats} / \mathrm{sec}$. This type of beating usually lasted for 2-10 min. The end of the bending motion often coincided with a further split of the axonemal portion into several parts, which proceeded explosively. We noticed that a special bundle of microtubules in an extensively frayed axoneme

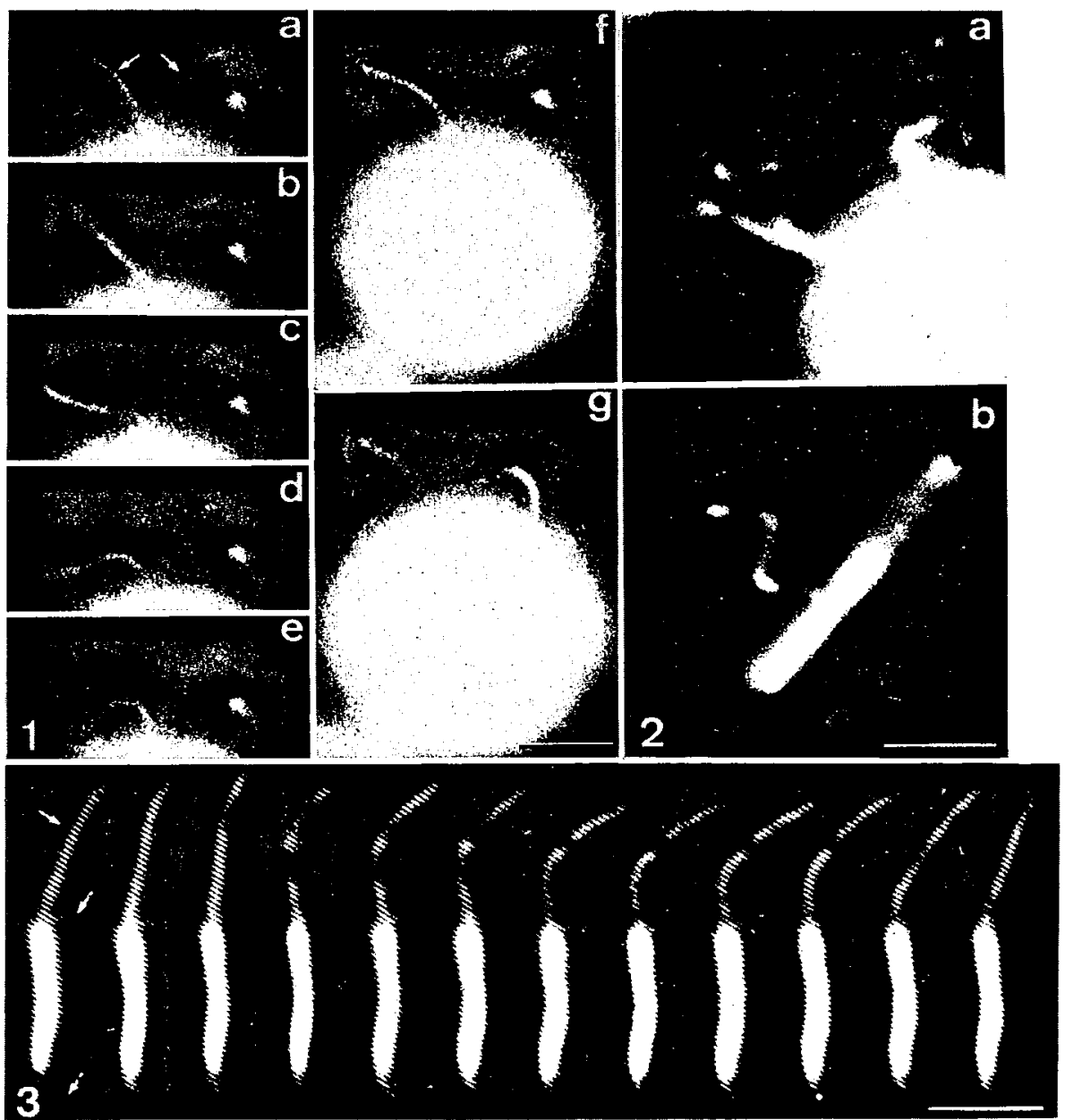

Fig. 1. A series of cinematographic frames showing the bending of a split fiber of an axoneme. One of the two flagella has split into two fibers as indicated by the arrows. From (a) to (f), the time interval between adjacent frames was $1 / 8 \mathrm{sec}$. In $(\mathrm{g})$ the focusing was varied so as to see the other flagellum, which had not split. The bar is $5 \mu \mathrm{m}$.

Fig. 2. (a), (b), A helical fiber in frayed axonemes. The bar is $5 \mu \mathrm{m}$.

Fig. 3. A TV recording of a partially disintegrated axoneme. Arrows indicate bundles of microtubules extruding from the ends of the axoneme. The time interval between two adjacent frames was $1 / 12 \mathrm{sec}$, the right one being later. The bar is $5 \mu \mathrm{m}$. 
had a lefthanded helical configuration with a pitch of $3.8 \mu \mathrm{m}$ and a diameter of 2.5 $\mu \mathrm{m}$, while other bundles had much smaller curvatures (Fig. 2). The gross morphology of this helix was discretely different from the close-coiled shape assumed by individual doublet microtubules of sperm axonemes (4). This unique helix was probably the pair of central singlet microtubules, because Hopkins (2), Allen and Borisy (1) and we (unpublished results) have observed in electron microscopy of negatively stained specimens that these paired microtubules exhibited a marked tendency to bend into an arc with a similar curvature. We sometimes observed that axonemes from which the putative central pair of microtubules had split off still showed bending motion. This observation is apparently incompatible with the report of Witman et al. (9), who found that flagella of Chlamydomonas which genetically lacked the central pair structure could not produce a bending motion.

It was not clear whether the whole length of an axoneme split from the tip to the proximal end, because the enormous brightness of the cell body hindered observation of the proximal region. If the proximal portion of the axoneme had not been split, then active sliding between the microtubules in this region could have moved the frayed tip. Possibly the above observations might thus have dealt with only passive movements. However, evidence for the ability of split axonemes to propagate bending waves came from observations of detached axonemes. When left in the presence of ATP and detergent, some free axonemes partially disintegrated through active sliding between the groups of microtubules, as has been observed in trypsinized axonemes $(8,9)$. Figure 3 is a TV recording of a free axoneme which has three bundles of microtubules extruding from both ends. This series of photographs clearly demonstrates that the longest bundle was propagating a bending wave from the tip (the proximal end) to the middle region. This wave propagation lasted for about $5 \mathrm{~min}$ with an almost constant frequency of $1.0 \mathrm{beat} / \mathrm{sec}$, but became slower and less regular within $10 \mathrm{~min}$.

We concluded that an assembly of a relatively small number of microtubules can exhibit oscillatory bending motion. We cannot yet tell what the minimum number of microtubules is that is required for the generation of bending. However, we have the impression that it must be less than four, since we often encountered cases where axonemes were frayed into three bundles, and two of them exhibited bending motion.

Acknowledgments. We thank Drs. S. Asakura and Y. Imae for their criticisms of our manuscript, and Dr. T. Ichimura for providing the strains of Chlamydomonas. This work was supported by a grant from the Ministry of Education, Science and Culture, of Japan (220612).

\section{REFERENCES}

1. Allen, C. and G.G. Borisy. Structural polarity and directional growth of microtubules of Chlamydomonas flagella. J. Mol. Biol. 90, 381-402, 1974

2. Hopkins, J.M. Subsidiary components of the flagella of Chlamydomonas reinhardii. J. Cell Sci. 7, 823-839, 1970

3. Kamiya, R. and S. Asakura. Helical transformations of Salmonella flagella in vitro. J. Mol. Biol. 106, 167-186, 1976

4. Miki-Noumura, T. and R. Kamiya. Shape of microtubules in solutions. Exp. Cell Res. 97, 451-453, 1976

5. SAger, R. and S. Granick. Nutrisional studies with Chlamydomonas reinhardi. Ann. N.Y. Acad. Sci. 56, 831-838, 1953

6. SAtir, P.J. Studies on cilia. III. Further studies on the cilium tip and a "sliding filament" 
model of ciliary motility. J. Cell Biol. 39, 77-94, 1968

7. Shingyoji, C., A. Murakami and K. TAKAhAShi. Local reactivation of Triton-extracted flagella by iontophoretic application of ATP. Nature 265, 269-270, 1977

8. Summers, K.E. and I.R. Gibbons. Adenosine triphosphate-induced sliding of tubules in trypsintreated flagella of sea-urchin sperm. Proc. Natl. Acad. Sci. USA. 68, 3092-3096, 1971

9. Witman, G.B., R. Fay and J. Plummer. Chlamydomonas mutants: evidence for the roles of specific axonemal components in flagellar movement. In Cell Motility, Vol. 3, ed. Goldman, R., T. Pollard and J.L. Rosenbaum, Cold Spring Harbor Laboratory, Cold Spring Harbor, New York, pp. 969-986, 1976 